\title{
Group Label
}

National Cancer Institute

\section{Source}

National Cancer Institute. Group Label. NCI Thesaurus. Code C90391.

Character(s) assigned to identify a particular collection of subjects or ideas. 